the past 3 decades. We investigated if Japanese geographic and socioeconomic disparities in life expectancy at birth (LE) have widened in this period.

Methods We used data on the LE of municipalities calculated every 5 years between 1985 and 2005. The municipality is the smallest administrative unit, for which LE data are available. Sample sizes varied from 1963 to 3354 across years due to nationwide municipality mergers undertaken after 2000. We also gathered information on the unemployment rates and other socio-demographic characteristics of municipalities. We used the relative index of inequality (RII) of LE (which corresponds closely to the relative LE gap between the top vs bottom municipalities in terms of LE or socioeconomic status, accounting for the variations in population size across all municipalities). Results Among men, in 1985, the LE gap was 4.2\% when municipalities were ranked by LE and $1.6 \%$ when ranked by the unemployment rate (as ordered from the lowest to the highest). Among women, these gaps were $2.6 \%$ and $0.4 \%$, respectively. These values changed only slightly over time, showing a tendency for a slight increase among men after 2000 and a decrease in women after 1995. Conclusion In Japan, during the period 1985-2005 geographic and socioeconomic disparities in municipal LE were larger in men than women. However, the LE disparity has been relatively small and stable despite the increase in income inequality since the 1980s.

\section{P1-459 A WITHIN-HOST NETWORK OF HUMAN COINFECTION}

doi:10.1136/jech.2011.142976g.48

${ }^{1} E_{\text {Griffiths, }}{ }^{* 2} \mathrm{~A}$ Pedersen, ${ }^{3} \mathrm{~A}$ Fenton, ${ }^{1} 0$ Petchey. ${ }^{1}$ University of Sheffield, Sheffield, UK; ${ }^{2}$ University of Edinburgh, Edinburgh, UK; ${ }^{3}$ University of Liverpool, Liverpool, UK

Coinfection by multiple parasites affects human health, parasite dynamics and the efficacy of infectious disease prevention and treatment. The capacity for different parasites (viruses, bacteria, fungi, protozoa, helminths) to interact is poorly understood. Interspecific interactions between coinfecting parasites could occur in many ways, either directly or indirectly with the host's immune system or bodily resources. We aimed to summarise connected resources, parasites and immune system components in coinfected humans using a network approach. The published literature contains thousands of records of coinfections in humans, associated immune responses, as well as parasite resource requirements. We used over 250 publications on human coinfection from 2009 to build an evidence-based parasite-immune system-resource network. We recorded the identity of coinfecting parasites, immune system components, host resources and the reported relationships between them. The network represents the potential for parasites to interact based on observation and theory found in recent coinfection literature. Results show the great taxonomic variety of coinfecting parasites, with particular involvement of viruses. Some parasites were reported in more coinfections, most notably HIV, suggesting that recent disease invasion and induced immunodeficiency may facilitate many parasite interactions. The network can also be used to generate numerous hypotheses for modelling work and suggestions for future observational and experimental research. The use of networks and other research tools to understand parasite interactions within coinfected hosts will help predict the potential for and consequences of disease invasions, as well as improve infectious disease interventions.

\section{P1-460 MOTHER MIGRATION AND IMMUNISATION STATUS OF CHILDREN ACROSS DIVERSE SETTINGS IN INDIA}

doi:10.1136/jech.2011.142976g.49

A Kumar, ${ }^{*}$ V K Singh. Banaras Hindu University, Varanasi, Uttar Pradesh, India

Introduction The geographical, cultural and socio-economic diversity of India implies that coverage of immunisation programmes and uptake varies between rural and urban areas, among different geographical regions and states. It has been seen that mother's migration is an important determinant of child immunisation uptake. This study examines the individual and community level explanatory factors associated with child immunisation differentials between migrant and non-migrant mothers groups in two states that is, Uttar Pradesh (UP) and Kerala of India. These two Indian states one in South (Kerala, where immunisation coverage is about $80 \%$ ) and another in North (UP- immunisation coverage is below $50 \%$ ) have different socio-economic, demographic and cultural characteristics.

Methods The data from the National Family Health Survey (NFHS-3) has been used in this study. The study is limited to children born during the 59 months before the interview. Multiple logistic regression analyses have been carried out to assess the relative contribution of independent variables on immunisation status.

Results The results indicate that Individual and community level variables are strongly associated with the likelihood of receiving full immunisation among migrant groups. The likelihood of full immunisation was higher for children of urban non-migrant mothers compared to children of rural-urban migrant mothers in UP while in Kerala, the vice-versa is true.

Conclusion Even after the enormous efforts by the government to popularise childhood immunisation, the lack of awareness among the parents, especially the mothers, remained a dominant reason for not vaccinating the child.

\section{P1-461 STEPS IN ASSESSING THE HEALTH OF A COMMUNITY: THE HEALTHY COMMUNITY COUNCIL 2011 ASSESSMENT PROCESS}

doi:10.1136/jech.2011.142976g.50

${ }^{1} \mathrm{~K}$ Lewis, ${ }^{*}{ }^{1,2} \mathrm{C}$ Reeves. ${ }^{1} J a m e s$ Madison University, Harrisonburg, Virginia, USA; ${ }^{2}$ Rockingham Memorial Hospital, Harrisonburg, Virginia, USA

The Harrisonburg-Rockingham Healthy Community Council (HCC) is a community-based organization located in the Shenandoah Valley of Virginia that was established in 1995 with the mission "to enhance the quality of life for the community through collaborative efforts of individuals, agencies, and institutions." To accomplish this mission, HCC members have conducted community needs assessments on quality of life in 1996, 2001, and 2006. Data from each assessment has been utilised to determine community need. Previous assessments have led to over $\$ 11$ million in funding for community initiatives. The HCC has just implemented the process for conducting the 2011 community needs assessment. Emphasis will be on the comparison of previous assessment data from 2001 to 2006 to evaluate longitudinal trends. The objective of this presentation is to provide a detailed step-bystep process for a comprehensive community needs assessment. Steps to conducting the 2006 and 2011 assessment include (1) forming a community needs coalition, (2) selecting communityspecific indicators, (3) developing a community needs assessment instrument, (4) obtaining a random sample of assessment participants, (5) collecting, managing, and analysing assessment data, (6) utilising resources (budget, in-kind contributions, etc) throughout the assessment process, (7) interpreting assessment data, (8) ascertaining local, state, national and international data for comparison purposes, (9) tying outcomes to assessment data, and (10) finalising a written report. The steps can be utilised by any agency or organization to develop a community-specific assessment to identify needs and establish priorities. Specific techniques including common pit falls to conducting community assessments will be addressed. 